\title{
Research the Optimal Path of Post-Disaster Relief Vehicles Based on Ant Colony Algorithm and WSN
}

\author{
Gao Weimin \\ School of computer and information science, Hunan institute of technology, Heng \\ yang 421002, China \\ E-mail:gwmhy@163.com
}

\begin{abstract}
Choosing a reasonable Rescue route guarantees emergency rescue command center to start the rescue work timely and effectively. In this paper, use the optimal path choice of public emergencies as an entry point of research build a post-disaster Ad Hoc net through wireless sensor technology, use the limited sensor resources to meet the rescue vehicle path optimization problem. Ant Colony Algorithm absorb the behavior of insect kingdom ant, use internal search mechanism to introduce sensor networks useful information, Fully consider the impact of the road traffic rescue vehicle travel time because of changes the traffic capacity, from rescue vehicles transport resources' two factors -the timeliness and economy, maximize meet the transport requirements of post-disaster emergency relief material to reduce the aid does not lead to timely the direct and indirect losses, maximize meet the transport requirements of post-disaster emergency relief material to reduce the aid does not lead to timely the direct and indirect losses. The simulation results show that the design of the optimal path to solve the disaster relief vehicles have obvious advantages and important practical significance.It Improved ant colony algorithm can quickly build a optimal routing, improve the efficiency of path optimization, effectively reduce the node energy consumption and ensure the whole network energy balance.
\end{abstract}

Keywords: ant colony algorithm, wireless sensor network, rescue vehicle, optimal path

\section{Introduction}

Earthquakes, fires, tsunamis, landslides and other natural disasters will destroy original local communication based fixed infrastructure, which lead to an overall communication interruption. The paralysis of communication infrastructure will lead that the disaster cannot be heard in a timely manner and influence the relief of the disaster. Mobile Ad Hoc Networks has many advantages- fast and flexible networking, no need to set up the network infrastructure and strong system survivability, which is the best choice for the deployment of disaster relief communication systems.

Ant colony algorithm is the latest bionic optimization to analog insect kingdom intelligent ant colony behavior. The ants have an ability to find the shortest path between the nest and food by leaving it in volatile secretions in past ways. Ants would leave the volatile pheromones at a way forward. Later, ants choose the path and the probability of the strength of the material on this path was proportional. For a path, the more ants choose it, the strong pheromone the ants leave in the path. Strong pheromone will attract more ants. This will cause a positive feedback. Finally, the ants can find the shortest path through the feedback.

When the ants solve the path selection problem, scholars mainly through computer simulations and mathematical modeling methods. Hadas. Y [1] considered under different types of accidents, such as safety, fires and traffic accidents. They will choose emergency vehicle routing algorithm and the factors influencing. On the base of dynamic traffic, we 
can achieve an emergency vehicle selection through $\mathrm{k}$ shortest path algorithm and simulation.

Hall [2] studied and analyzed Random time-dependent network. But he didn't solve its dependency through specific algorithm. Research shows the desired path and the shortest path in the form of the probability distribution function. It has a certain reference value. Loui [3] By finding the shortest path algorithm optimization theory has some limitations. This limits its use in general non-linear utility function in question. It is also desirable to maximize the utility of the method also has a problem. Using it to solve the random network optimal path is inappropriate. Bard [4] thinks that the transport system has two main features after the earthquake. Changes in traffic demand and network damage concreteness. But Monte Carlo simulation algorithm of his research, combined with Network Path randomly selected and utility function and other methods to solve nonlinear path selection utility functions shortest path problem. Yang [5] rescue vehicles, taking into account changing conditions varying characteristics disaster scheduling, establishing a dynamic network planning model for emergency vehicle scheduling. Yi and Kumar [6] to one kind of emergency vehicle scheduling problem into more goods distribution and vehicle routing two sub-problems, network flow model is constructed in stages.

Ant Colony Algorithm for domestic research started late.Until 1997, Our only appeared published a paper on the ant colony algorithm. Since the ant colony algorithm is introduced into China, after the theoretical study of ant colony algorithm gradually up. It is mainly about to improve ant colony system model, optimal combination of parameters set research. The ant colony optimization theory to solve practical problems in recently years. But it needs to be further research applications, having great use of space.

\section{Rescue the Optimal Path Mathematical Model of Ant Colony Algorithm}

Supposed $m$ was the amount of ants, $n$ was the number of nodes, $C_{i}(t)$ represent the number of ants on node $\mathrm{i}$ at moment $\mathrm{t}$, thus the number of ant colonies $m=\sum_{1}^{n} c_{i}(t) ; \mathrm{V}$ represent the assemblage of $\mathrm{n}$ nodes, $\mathrm{E}$ represents the assemblage of edges between each pair of nodes, $\xi_{\mathrm{ij}}(\mathrm{t})$ represents the density of pheromone on path $<\mathrm{i}, \mathrm{j}>$ at moment $\mathrm{t}$ which passed through by ant colonies, $\xi=\left\{\xi_{i j}(t) \mid v_{i}, v_{j} \in v\right\}$ represent the assemblage of pheromone level on path $\langle i, j\rangle$ which connected any pair of nodes in assemblage $v$ at moment t. Generally, solving optimum path matter based on ant colony algorithm can be done with the digraph $G=(v, E, \xi)$ by acquiring the minimum weight.

In the process of ant $\mathrm{K}(\mathrm{K}=1,2, \ldots, \mathrm{m})$ 's movement,the pheromone of each paths will affect their movement direction. Provided we use taboo table tabu $\mathrm{k}_{\mathrm{k}}(\mathrm{k}=1,2, \ldots, \mathrm{m})$ to record the nodes that ant $\mathrm{K}$ walked, path assemblage will make dynamic adjustment according to $t_{a b u}$. When choosing the path, ant will calculate the condition transfer probability according to the pheromone level of each path and heuristic information. Given $p_{i j}^{k}(t)$ represent the condition transfer possibility of ant $\mathrm{K}$ moving from node $\mathrm{i}$ to node $\mathrm{j}$ at moment t:

$$
\begin{gathered}
p_{i j}^{k}(t)=\frac{\left[\xi_{i j}(t)\right]^{\alpha}\left[\eta_{i j}(t)\right]^{\beta}}{\sum_{\text {seallowed }_{k}}\left[\xi_{i s}(t)\right]^{\alpha}\left[\eta_{i s}[t]\right]^{\beta}} \text { if } j \in \text { allowed }_{k} \\
0 \quad \text { if } j \notin \text { allowed }_{k}
\end{gathered}
$$

In this equation, allowed $=\left\{V-{ }^{\text {tabu }}{ }^{u_{k}}\right\}$ represent the nodes that ant $\mathrm{K}$ was permitted to choose the next step; $\alpha$ represents heuristic information factor which is used to embody 
the relative importance of pheromone, $\beta$ represents heuristic expectation factor; $\eta_{i j}(t)$ represents heuristic function which means the expectation level of ant $\mathrm{K}$ moving from node $\mathrm{i}$ to node $\mathrm{j}, \eta_{i j}=\frac{1}{d_{i j}} \cdot d_{i j}$ represents the distance of two mutual neighbor nodes $\mathrm{i}, \mathrm{j}$, the path $\left\langle\mathrm{i}, \mathrm{j}>\right.$. As for ant $\mathrm{K}$, the shorter $\mathrm{d}_{\mathrm{ij}}$, the higher $\eta_{i j}(t)$ and $p_{i j}^{k}(t)$ will be.

After each ant moved one step or finished the traversal of all $n$ nodes, remained information will be updated. As we mentioned above, we use $\xi_{i j}(t)$ represent the pheromone level of path $\langle i, j\rangle$ passed by ant $\mathrm{K}$ at moment $\mathrm{t}$, therefore the pheromone level of path $\langle\mathrm{i}, \mathrm{j}\rangle$ at moment $\mathrm{t}+1$ will be $\xi_{i j}(t+1)=(1-\rho) \xi_{i j}(t)+\sum_{k=1}^{m} \Delta \xi_{i j}^{k}(t)$, in this formula, $\rho$ represents pheromone volatilize coefficient, $\rho \in[0,1]$, thus $\rho-1$ represents pheromone level remain factor; $\Delta \xi_{i j}^{k}(t)$ represents the increment of pheromone level of ant $\mathrm{K}$ from moment $t$ to moment $t+1$ on path $\langle i, j\rangle$.Because of the differences of pheromone update strategies, the way of calculating $\Delta \xi_{i j}^{k}(t)$ will varies greatly in different ant colony algorithm models.

\section{The Adaptive Ant Colony Algorithm based on WSN}

Wireless Sensor Network Consists of many sensor nodes can communicate with each other constitute. As shown in figure 1, These nodes can be for the information of the object sensing area is to collaborate monitoring, sensing, collecting. And then these information processing, and by Remote Task Manager will process the results transmitted to the information needs of these users.

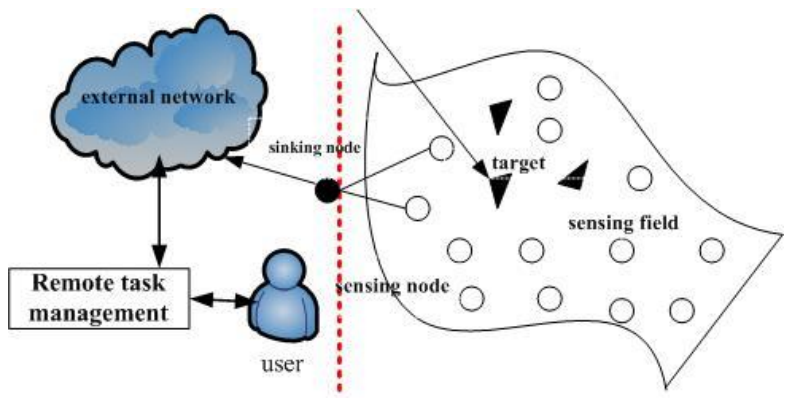

Figure 1 WSN System Constitute

Ant colony optimization algorithm path contains stage adaptation phase and collaboration. In the adaptation phase, each candidate solution is continually adjusting its structure based on the accumulated information. The larger amount of information, the path more likely to be selected, and the longer time will be spent. In collaboration stage, through the exchange of information between the candidate solution to expect to yield better performance solution. Basic ant colony algorithm is based on the principles of positive feedback information and the combination of heuristics. But with some shortcomings, mainly expressing that Heuristic solution in the course of construction, due to the random selection policy algorithm slow evolution; and the positive feedback principle is intended to strengthen the performance of the solution is better, but prone to stagnation.

In response to these deficiencies, to improve research from the selection strategy, we randomly selected deterministic selection and combination of selection policy first. Key 
areas required to take samples to detect the use of WSN data, and dynamically during the search probability for certainty selected adjustment. When the evolution to a certain algebra, the direction of evolution has been basically established, then the amount of information on the path as dynamic adjustment, narrow the best and worst path information gap and appropriate to increase the probability of randomly selected, in order to facilitate the solution space more complete search, thereby to effectively overcome the basic ant colony algorithm of two problems, the improved method belongs to adaptive methods.

\section{Rescue Vehicle Scheduling and Optimal Path Analysis}

\subsection{Analysis of Characteristics of Rescue Vehicles Scheduling}

Emergency rescue vehicles have the following characteristics

(1) Urgency. Since the emergency rescue are due to emergencies caused by the objective requirements of the vehicle scheduling decisions quickly and effectively, in order to ensure the completion of the rescue mission.

(2) Uncertainty and variability. Time and place of emergencies cannot be foreknowing. And it is difficult to predict its changes. In the early stage of emergency response, the demand and supply of all kinds of relief supplies and rescue workers can be calculated accurately. The psychological and behavioral characteristics of people in the event of unexpected incidents are not certain.

(3) Weak Economy. Incident to the basic values and code of conduct of the social system architecture serious threat, In the emergency response should first control the situation as much as possible to spread and reduce casualties, restore social order as soon as possible and other non-economic objectives is often the most important goal of the rescue vehicle scheduling. While the costs, benefits and other economic goals are not the only target.

(4) Limited resources. After the incident occurred, the need for relief supplies and rescue workers surge in a short time usually does not have enough resources to fully meet these needs.

\subsection{Rescue Vehicles Scheduling Process}

The post-disaster relief vehicles scheduling process is shown in figure 2 .

Location of service point to disaster relief. After the disaster, disaster emergency rescue service is the most important work. Rescue service point site should follow the following principles:

(1)Disaster relief services and information and road points should be connected to the command center kept open;

(2) Disaster relief service points should be selected in place of secondary disasters do not occur, and the victims in the affected areas within easy reach;

(3) Disaster relief service point should be able to accommodate a certain number of victims.

Stricken path information: In determining the location and disaster relief command center after the rescue service points, available information to determine the optimal path of emergency supplies distribution centers and disaster relief programs and services based on the real-time path between points. To enable emergency supplies to reach the emergency location quickly and with as little cost to reach the rescue service locations, When determining a scheduling program generally have the shortest transportation and distribution of most of the cost of two objective functions. The two objective functions, duration of emergency material dispatching are often directly related to rescue the success or not, so the transportation time shortest as the main objective functions. 


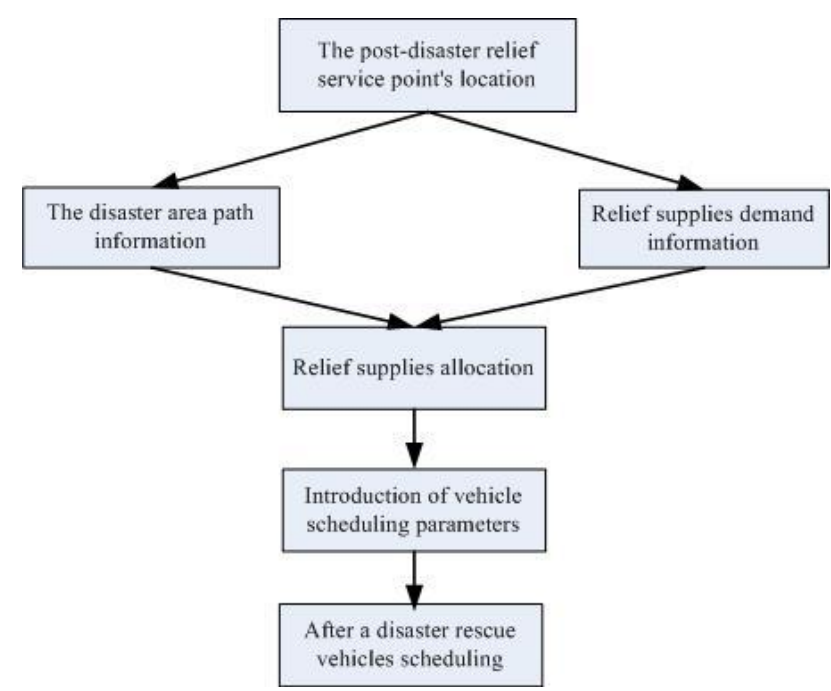

Figure 2. The Post-disaster Relief Vehicles Scheduling Process

Real-time vehicle scheduling parameters: During disaster relief vehicle scheduling, vehicle scheduling parameters to be set according to the scheduling requirements. Mainly lies:

(1) Disaster relief restrictions. That is the best time to rescue the urgency of prime time and meet other conditions. If, after the earthquake disaster, effectively saving lives is the best time within 72 hours, in this limited period of time for efficient and orderly rescue operations, in order to reduce the losses caused by disasters to a minimum.

(2) Priority relief supplies. A priority relief supply is also essential to determine scheduling scheme. It directly affects the design of scheduling scheme. Different Loss aid does not meet the requirements of the time caused by the extent of the loss and the importance of materials, degree of timeliness and gaps and other relevant. For after the disaster, the injured should an emergency rescue in the first place, the load transport vehicles, the number of vehicles can be put into the scheduling parameters and the various rescue services facility emergency supplies scheduling demand for emergency supplies and other needs are determined in advance.

\subsection{Based on ACA and WSN to Solve the Optimal Path}

Based on the basic idea of ACA and WSN disaster rescue vehicles for optimal path: Firstly, the optimal path data model WSN. Then according to vehicle scheduling parameters for data transmission route to initialize. Then adaptive ant colony optimization algorithm path. In each round, the process of optimization, genetic algorithm for improved algorithm to obtain the solution ACA selection, crossover and mutation operations to improve the efficiency and speed of solving. Finally, wireless sensor network detection output in the optimal path road disaster relief vehicles.

Its working process is shown in figure 3. 


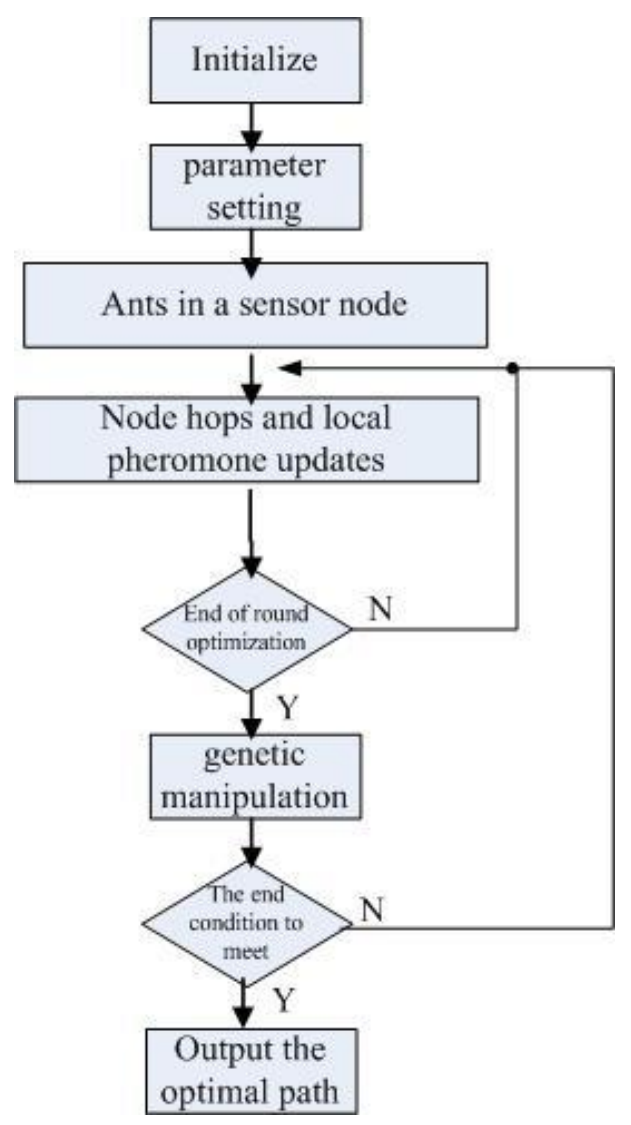

Figure 3. The Optimal Path Solving Process

\section{Simulation Study}

In order to test adaptive ant colony algorithm based WSN optimal path for solving performance, the use of network simulation software NS2 build simulation testing laboratories.

Figure 4 is 11 affected points after some county in the affected area map, the emergency rescue center located in 0 points, emergency supplies from here are transferred to the disaster relief stations for point. To rescue some demand of emergency supplies are shown in table 1.

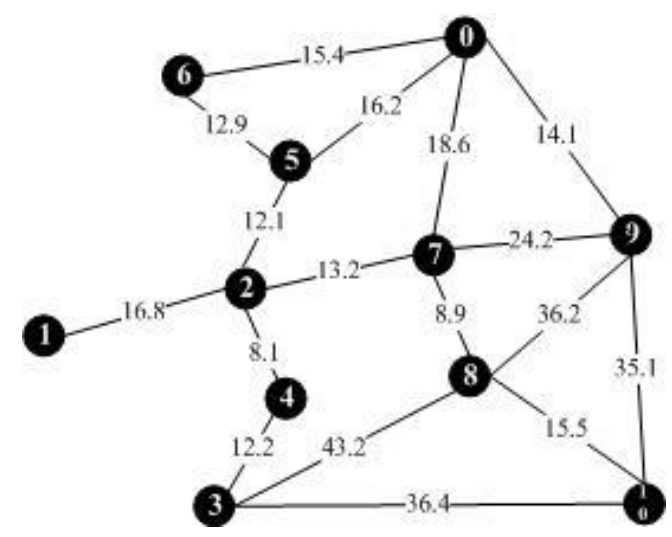

Figure 4. Some County in the Affected Area Map

Table 1 Demand for Emergency Supplies of the Affected Areas 


\begin{tabular}{|l|l|l|l|l|l|l|l|l|l|l|}
\hline $\begin{array}{l}\text { Affected } \\
\text { areas }\end{array}$ & 10 & 9 & 8 & 7 & 6 & 5 & 4 & 3 & 2 & 1 \\
\hline $\begin{array}{l}\text { Material } \\
\text { quantity }\end{array}$ & 2.6 & 2.2 & 3.1 & 4.4 & 1.3 & 0.9 & 1.8 & 1.3 & 2.5 & 1.2 \\
\hline
\end{tabular}

In order to save the cost of transport of emergency relief supplies. Taking into account the demand for emergency supplies all the affected areas are less than truck load, in ensuring the shortest possible response time requirements of condition, relief supplies to each of the affected spot needs as much as possible with only a trucking.

Simulation experiments are carried out in three cases:

(1) Without taking into account the winding and direct connection of the road, the optimal path is carried out only from the diagram of the road network and the location coordinates of the AcA algorithm. The optimal path of the emergency rescue vehicle is as follows, the shortest total distance: $187.9 \mathrm{~km}$.

$0 \rightarrow 1 \rightarrow 2 \rightarrow 3 \rightarrow 7 \rightarrow 6 \rightarrow 0$

$0 \rightarrow 4 \rightarrow 9 \rightarrow 10 \rightarrow 8 \rightarrow 0$

$0 \rightarrow 5 \rightarrow 0$

(2) If the path of the road is considered as well as the direct connectivity, but the path weights are not considered, the ant colony parameters are set up as above. The results are as follows:

$0 \rightarrow 2 \rightarrow 3 \rightarrow 10 \rightarrow 0$

$0 \rightarrow 4 \rightarrow 8 \rightarrow 9 \rightarrow 5 \rightarrow 0$

$0 \rightarrow 6 \rightarrow 7 \rightarrow 0$

The shortest path total distance of the emergency rescue vehicle is $268.5 \mathrm{~km}$.

(3) In the process of solving the optimal path, the path weight matrix is introduced into the algorithm by using the data of WSN, which is based on the winding, direct connectivity and path weight of the road. Ant colony parameters set up as above, the simulation results are as follows.

$0 \rightarrow 2 \rightarrow 3 \rightarrow 7 \rightarrow 6 \rightarrow 0$

$0 \rightarrow 10 \rightarrow 11 \rightarrow 9 \rightarrow 4 \rightarrow 0$

$0 \rightarrow 5 \rightarrow 8 \rightarrow 0$

The shortest path total distance of the emergency rescue vehicle is $279.1 \mathrm{~km}$.

In three cases, the optimal path hop count comparison and AcA algorithm and the improved ant colony algorithm and WSN algorithm energy consumption are shown in Figure 5.

After the introduction of the path weight matrix, although the shortest total distance transport increased, it takes into account some of the actual situation such as road grade, road curvature, slope and traffic, the time required to complete the distribution of emergency supplies task less. It means that optimization algorithm highest efficiency to quickly establish the optimal path WSN, which has a more important role in the emergency response optimal path selection.
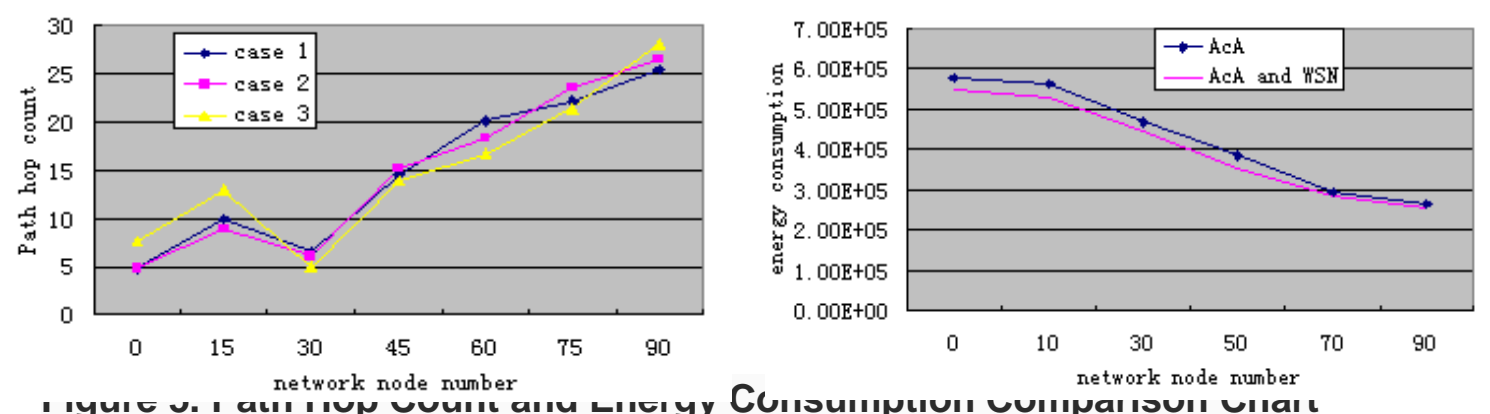

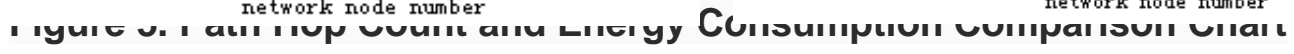




\section{Conclusion}

As to Genetic algorithm and ant colony optimization algorithm in path shortcomings, the use of their advantages, the genetic manipulation of genetic algorithm ant colony algorithm is introduced to the path optimization process, improving ant colony algorithm. The simulation results show that improved ant colony algorithm to quickly build an optimal routing, route optimization to improve efficiency, reduce energy consumption of nodes, ensure that the entire network energy balance, improve network reliability and adaptability.

\section{Acknowledgment}

This work was financially supported by Ministry of education of humanities and social science research fund (No.15YJC880017); Hunan province office of education planning issues (No.XJK014CXX004); Philosophy and social science foundation in Hunan province (No.14YBA129)

\section{References}

[1] Gaoweimin, "Problem of Shortest Path in Bus Net Based on Ant Algorithm", Computer and Communications, vol. 25, no. 134, (2007), pp. 94-103.

[2] Y. Hadas and A. Ceder, "Shortest Path of Emergency Vehicles under Uncertain Urban Traffic Conditions", Transportation Research Record, vol. 14, no. 12, (1996), pp. 34-39.

[3] R. W. Hall, "The fastest path through a network with random time-dependent travel Times", Transportation Science, vol. 6, no. 12, (2009), pp. 148-156.

[4] R. Loui, "Optimal path in graphs with stochastic or mudding tensional weights", Communications of the ACM, vol. 11, no. 4, (1999), pp. 64-82.

[5] N. Basoz, M. Williams and A. Kiremidjian, "A Gis-Based Emergency Response System for Transportation Networks", Earthquake Engineering, vol. 15, no. 10, (2003), pp. 121-131.

[6] S. Yang, H. Masoud and A. Haghani, "Online Dispatching and Routing Model for Emergency Vehicles with Area Coverage Constraints", Transportation Research Record, vol. 3, no. 6, (2005), pp. $1-8$.

[7] W. Yi and A. Kumar, "Ant Colony Optimization for Disaster Relief Operations", Transportation Research, vol. 10, no. 1, (2008), pp. 660-672.

[8] L. Yong, "Research on the optimal path of emergency rescue based on ant colony algorithm", China University of Geo-sciences:Wuhan, (2010).

[9] Z. Yaming, S. Haoshan, L. Yan and J. Fei, "A Better Itinerary Analysis for Mobile Agent(MA) through Using ACA-SAA Algorithm in Wireless Sensor Networks", Journal of Northwestern polytechnical university, vol. 30, no. 5, (2012), pp. 329-634.

[10] S. Jin, Z. Qiuhong and Y. Xinfeng, "Path optimization of wireless sensor network based on improved ant colony algorithm".

\section{Authors}

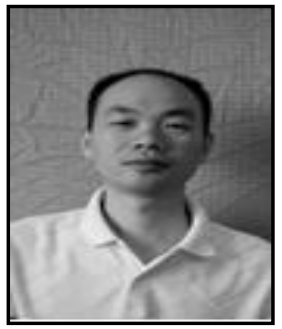

Weimin Gao, he is from the Hunan Province Qidong country, Associate Professor, School of Computer Science Master, Hunan Institute of Technology, mainly engaged in the research of Computer Network and Information Security. He has received Master degree from Hunan University, and his major is electronic engineering. His research interests include wireless sensor network, Computer Network and Information Security. 